\title{
EFFECTIVELY SIMPLE SETS
}

\section{RAYMOND M. SMULLYAN ${ }^{1}$}

We use the word "number" to mean positive integer, and "set" to mean set of positive integers. A set $\alpha$ is called simple (after Post [1]) iff $\alpha$ is r.e. (recursively enumerable), infinite, and its complement $\tilde{\alpha}$, though infinite, contains no infinite recursively enumerable subset. We shall call a set $\alpha$ effectively simple if $\alpha$ and its complement are infinite, $\alpha$ is r.e., and there exists a recursive function $\sigma(x)$ such that for any number $i$ for which $\omega_{i}$ is disjoint from $\alpha$, the number $\sigma(i)$ is greater than the number of elements of $\omega_{i \cdot}{ }^{2}$ Informally speaking, this means that any $\omega_{i}$ disjoint from $\alpha$ is not only finite (which simply says that $\alpha$ is simple), but that we can effectively find a bound for the number of elements of $\omega_{i}$.

The original simple set $S$ constructed by Post [1] is effectively simple, indeed it is immediate from Post's argument that the function $\sigma(x)=2 x+1$ has the desired property. As is well known, the set $S$ is not hypersimple. Our present purpose is to prove the existence of an effectively simple set which is also hypersimple.

A number $i$ is said to be a deficiency point of a function $f(x)$ if there exists a number $j>i$ such that $f(j)<f(i)$. The set of all deficiency points of $f$ is called the deficiency set of $f$. Dekker [4] has shown that if $f$ is a 1-1 recursive function which enumerates a recursively enumerable but not recursive set, then the deficiency set of $f$ is simple, in fact hypersimple. We show

THEOREM. Let $f$ be a 1-1 recursive function which enumerates a creative set $C$. Then the deficiency set $D$ of $f$ is effectively simple.

In the above theorem, the set $D$ is hypersimple (by Dekker's theorem), so we obtain a hypersimple set which is effectively simple.

Proof of Theorem. For any set $A$, let $A^{*}$ be the set of all numbers $x$ such that for some element $y$ of $A, x<f(y)$, but $x \neq f(w)$ for every

Received by the editors November 10,1962 and, in revised form, April 20, 1963.

1 The research in this paper was supported in part by a grant from the Air Force Office of Scientific Research.

2 We use the recursive enumeration $\omega_{1}, \omega_{2}, \cdots, \omega_{i}, \cdots$ of all r.e. sets, as defined in [2]. There we used a predicate $T_{1}(x, y, z)$, substantially that of Kleene [3], except that the variables range over positive integers only. Thus $\omega_{i}$ is the set of all $x$ 's satisfying the condition: $(\exists y) T_{1}(i, x, y)$. Our restriction of r.e. sets to sets of positive integers follows the lines of Post [1] rather than Kleene [3]. Of course, we could easily modify our arguments, if we wish to allow 0 as a member of an r.e. set. 
$w \leqq y$. Thus $x \in A^{*} \leftrightarrow(\exists y) \quad\left[y \in A \wedge x<f(y) \wedge(\forall w)_{\leq y} x \neq f(w)\right]$. It is clear that if $A$ is r.e., so is $A^{*}$. Moreover given any index $i$ of $A$, we can effectively find an index of $A^{*}$; more precisely, there is a general recursive function $t(x)$ such that for every $i, \omega_{t(i)}=\omega_{i}^{*}$. This follows by the iteration theorem (cf. [2, p. 68, Theorem 2]), since the condition $(\exists y)\left[y \in \omega_{z} \wedge x \leqq f(y) \wedge(\forall w)_{\leq y} x \neq f(w)\right]$ is a recursively enumerable relation (of the 2 variables $x, z$ ).

We now show that if $\omega_{i}$ is disjoint from the deficiency set $D$ of $f$, then $\omega_{i}^{*}$ must be disjoint from $C$; equivalently, if $\omega_{i}^{*}$ has an element in common with $C$, then $\omega_{i}$ has an element in common with $D$. For suppose $\omega_{i}^{*}$ has an element in common with $C$. This element is of the form $f(z)$, since $C$ is the range of $f$. Thus $f(z) \in \omega_{i}^{*}$, so for some $y \in \omega_{i}$, $f(z) \leqq f(y)$, but $f(z) \neq f(w)$ for every $w \leqq y$. In particular, $f(z) \neq f(y)$. Hence $f(z)<f(y)$. Since $f(z) \neq f(w)$ for every $w \leqq y$, then $z \neq w$ for every $w \leqq y$ (because $f$ is $1-1$ ). This implies that $z>y$. Thus $f(z)<f(y)$, but $z>y$. Hence $y$ is a deficiency point of $f$, so must be in the set $D$. Thus $y$ is in both $\omega_{i}$ and $D$.

We next show that for any set $\omega_{i}$, if $a$ is any number outside both $C$ and $\omega_{i}^{*}$, then $a$ is greater than $f(y)$ for every $y \in \omega_{i}$. For take a number $a$ outside $C$ and $\omega_{i}^{*}$. Since $a \notin C$, then $a$ is not of the form $f(w)$. Since $a \notin \omega_{i}^{*}$, then for every $y \in \omega_{i}$, either $a>f(y)$, or $a=f(w)$ for some $w \leqq y$. The latter alternative cannot hold, since $a$ is not of the form $f(w)$. Hence $a>f(y)$.

The set $C$ is creative; let $\phi(x)$ be a productive function for the complement of $C$. We take the function $t(x)$ previously considered, and we assert that $\phi[t(x)]$ is the function we seek, i.e., if $\omega_{i}$ is disjoint from $D$, then $\phi[t(x)]$ must be an upper bound for the number of elements of $\omega_{i}$. The proof of this merely pieces together facts which we have already proved.

Suppose $\omega_{i}$ is disjoint from $D$. Then $\omega_{t(i)}$ is disjoint from $C$. Hence $\phi[t(i)]$ must be outside both $C$ and $\omega_{i}$ (because $\phi$ is a productive function for the complement of $C$ ). Hence $\phi[t(i)]$ is greater than $f(y)$ for every $y \in \omega_{i}$. Since $f$ is $1-1$, then $\phi[t(i)]$ must be greater than the number of elements of $\omega_{i}$. This concludes the proof.

REMARKs. Some time ago I posed the question to Gerald T. Sacks whether there exists any simple set which is not effectively simple. He has recently proved that there does, and has submitted this result to this journal.

\section{REFERENCES}

1. E. Post, Recursively enumerable sets of positive integers and their decision problems, Bull. Amer. Math. Soc. 50 (1944), 284-316. 
2. R. M. Smullyan, Theory of formal systems, Annals of Mathematics Studies No. 47, Princeton Univ. Press, Princeton, N. J., 1961.

3. S. C. Kleene, Introduction to metamathematics, Van Nostrand, New York, 1952.

4. J. C. E. Dekker, A theorem of hypersimple sets, Proc. Amer. Math. Soc. 5 (1954), 791-796.

YESHIVA UNIVERSITY

\section{ENTROPY FOR NONINVERTIBLE TRANSFORMATIONS}

\section{JOHN D. FERGUSON ${ }^{1}$}

1. Introduction. In 1959, Sinai (see [1]) gave an improved version of the definition of entropy for a measure-preserving transformation on a probability space. Included in the same paper was a theorem which made possible the computation of the entropy of certain invertible measure-preserving transformations. In this paper we prove a theorem, similar to that of Sinai, for measure-preserving transformations which are not necessarily invertible.

2. Preliminaries. Let $(X, S, \mu)$ be a probability space, and $T$ a measure-preserving transformation on $X$. If $\boldsymbol{A}$ and $\boldsymbol{C}$ are subfields of $\boldsymbol{S}$ with $\boldsymbol{A}$ finite, then the "entropy" and "conditional entropy" of $\boldsymbol{A}$, denoted $\bar{H}(\boldsymbol{A})$ and $\bar{H}(\boldsymbol{A} / \boldsymbol{C})$, respectively, are defined in Halmos [2]. Using these concepts, the entropy of $T$ is defined as follows. Let $\boldsymbol{A}$ be a finite subfield of $S$, then $\bigvee_{j=0}^{m} T^{-i} A$ is a finite subfield of $S$, and it follows from a theorem in information theory that

$$
h(T, \boldsymbol{A})=\lim _{m \rightarrow \infty}(1 /(m+1)) \bar{H}\left(\bigvee_{j=0}^{m} T^{-j} \boldsymbol{A}\right)
$$

exists. Then the entropy of $T$ is $h^{*}(T)$, where

$$
h^{*}(T)=\sup \{h(T, \boldsymbol{A}) \mid \boldsymbol{A} \text { a finite subfield of } \boldsymbol{S}\} .
$$

Sinai's essential idea was to avoid taking the supremum by exhibiting a finite subfield $\boldsymbol{A}$ of $\boldsymbol{S}$ for which $h(T, \boldsymbol{A})$ gave the supremum, but his proof depended on the invertibility of $T$. The theorem proved below replaces the supremum over all finite subfields $\boldsymbol{A}$ by a supre-

Received by the editors February 2, 1963 and, in revised form, August 1, 1963.

${ }^{1}$ Some of the contents of this paper were included in the author's doctoral dissertation, under the direction of Professor G. A. Hedlund, at Yale University, where the author held a National Science Foundation Graduate Fellowship. 